\title{
The Hybrid Classification Model Thanks to Artificial Neural Network and Artificial Immune Systems for Diagnosis of Epilepsy from Electroencephalography
}

\author{
Sema Arslan and Hakan Işik
}

\begin{abstract}
In this study, Artificial Neural Networks (ANN) and Artificial Immune (AI) techniques designed in the form of a hybrid structure are used for diagnosis of epilepsy patients via EEG signals. Attributes of EEG signals are needed to be determined by employing EEG signals which are recorded using EEG. In this process the raw digital signals data is received and is summarized in some respects. From this data, four characteristics are extracted for the classification process. $20 \%$ of available data is reserved for testing while $80 \%$ of available data is being reserved for training. These actions were repeated five times by performing cross-validation process. AIS is used for updating the weights during training ANN and a program is constituted for the classification of EEG signals. Education and recording processes were performed with different parameters by means of the constituted program. The obtained findings show that the proposed method was effective for achieving accurate results as much as possible with the use of ANN and AIS, together.
\end{abstract}

Index Terms-Artificial neural network, artificial immune systems, clonal selection, epilepsy, EEG signals.

\section{INTRODUCTION}

In the study of Sakthivel et al. (2011), compared the fault classification efficiency of AIRS with hybrid systems such as principle component analysis (PCA)-Naive Bayes and PCA-Bayes Net. It has been observed that the AIRS-based system outperforms the other two methods considered in the present study [1].

In this study of Zhao et al. (2011), proposed a new machine learning method for complex systems by integrating the AIS system with RBFPLS. This new method demonstrates its satisfactory effect on classification accuracy for clinical diagnosis, and also indicates its wide potential applications to other diagnosis and detection problems [2].

In the study of Guo et al. (2010), have used to multiclassify 5 kinds of mental tasks for Immune Feature Weighted SVM (IFWSVM). Theoretical analysis and experimental results show that IFWSVM has better performance than Immune SVM (ISVM) without feature weight [3].

In the study of Şengür et al. (2008), investigated the use

Manuscript received September 5, 2013; revised November 11, 2013.

Sema Arslan is with the Department of Computer Engineering, Faculty of Engineering and Architecture, Selcuk University (e-mail: semaarslan@ selcuk.edu.tr).

Hakan Issik is with the Department of Electronic and Computer Education, Faculty of Technical Education, Selcuk University, 42079, Konya-Turkey (e-mail: hisik@ selduk.edu.tr). of principal component analysis (PCA), artificial immune system (AIS) and fuzzy $k$-NN to determine the normal and abnormal heart valves from the Doppler heart sounds. The validation of the proposed method is measured by using the sensitivity and specificity parameters; $95.9 \%$ sensitivity and 96\% specificity rate was obtained [4].

In the study of Aickelinet et al. (2004), hoped that biologically inspired approaches in this area, including the use of immune-based systems will be able to meet this challenge. They collated the algorithms used, the development of the systems and the outcome of their implementation. [5].

In the study of Freitas et al. (2003), explained the potential pitfalls in representation selection and the use of various affinity measures. Then presents ideas on avoiding unnecessary mistakes in the choice and design of AIS algorithms and ultimately delivered solutions [6].

In the study of de Castro et al. (2002), presented the adaptation of an immune network model, originally proposed to perform information compression and data clustering, to solve multimodal function optimization problems [7].

In the study of de Hofmeyr et al. (2000), described an artificial immune which incorporates many properties of natural immune systems, including diversity, distributed computation, error tolerance, dynamic learning and adaptation, and self-monitoring [8].

In the study of de Coello et al. (2005), proposed an algorithm based on the clonal selection principle to solve multi-objective optimization problems (either constrained or unconstrained). Results indicate that the proposed approach is a viable alternative to solve multi-objective optimization problems [9].

\section{FORMUN ALTI-MATERIAL AND METHOD}

EEG signals are observed via vibrations of the electrical potentials (oscillations) that occur during brain activity through electrodes placed on the skull [10]. EEG signals are often referenced for understanding of the properties and the investigation of neurological and neuropsychological functions in the brain, today as well as for diagnostic and therapeutic purposes in many clinical [11].

\section{A. Data Selection}

In this study, EEG signals were taken from database described in Andrzejak et al. (2001). Feature vectors are removed using 4097 data obtained from epileptic patients and healthy individuals. Min, max, average and standard deviation is carried out for extracting this feature vectors. 
Thus, 200 data has obtained consisting of four feature vector.

From the obtained data, $20 \%$ was reserved for testing and $80 \%$ for training and these operations were repeated by 5 times by applying cross-validation.

\section{B. Artificial Neural Networks Models}

Artificial neural networks, modeled in the shape of the human brain to learn a method to try. An artificial neural network, called neurons, information processing consists of the elements. Neurons, sending a signal via the connection lines affect each other. These connecting lines each have a unique weight. These weights are calculated in the adaptive, information, reports the. Typically, the connection weights are determined by a learning process. Different weights can also be determined by the learning process is achieved by using the outcome information [12].

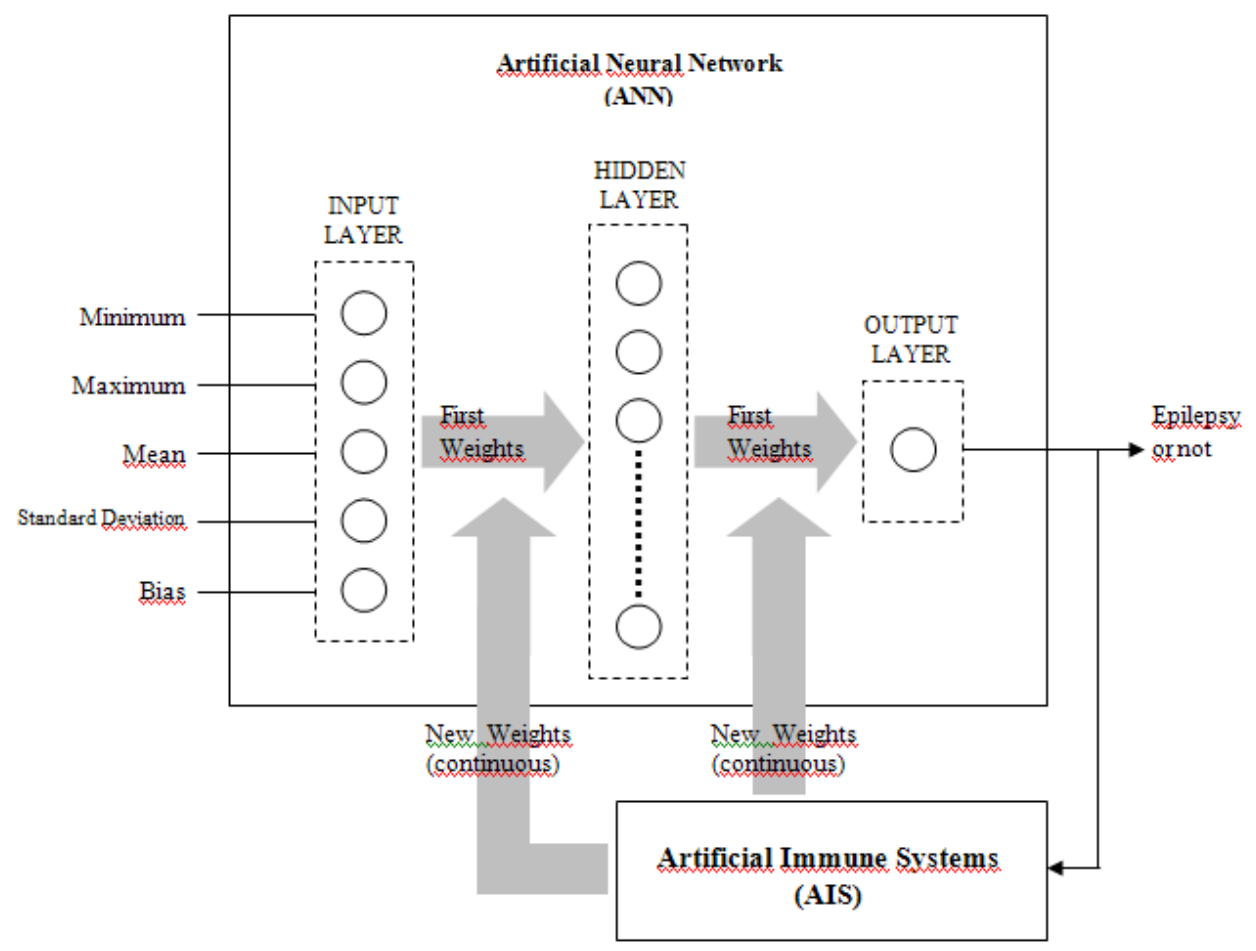

Fig. 1. Back-Propagation Multilayered Neural Network Structure with AIS.

\section{Artificial Immune Systems}

AIS; a complex model is applied to problem areas, in principle, observed immune functions and an adaptive system that was developed inspired by theoretical immunology [13]. The clonal selection algorithm, the immune system against an antigenic stimulus is used to describe the basic features. Recognize antigens only brings up the idea of these cells are selected in order to reproduce. The selected cells are subjected to the process of mutation enhances the similarities to the selective antigens [14].

\section{DESIGN OF THE HYBRID MODEL}

AIS is used for updating the weights after finding the value of the output error in ANN model. Thus, a hybrid model was carried out with AIS. Fig. 1 denotes the structure of hybrid model with back-propagation neural network and AIS.

The network input consists of four feature vector and a bias. The output value is the state of presence or absence of epilepsy. Input values and hidden layer neuron number of the network consisting of one hidden layer determine the vector space $(\mathrm{N})$ of AIS. The number of AI antibody changes depending on the number of neurons in hidden layer. In this model, the initial weight values are weights which are used in ANN. ANN and AIS are used in a hybrid structure for diagnosis of epilepsy disease. Sigmoid function is used to detect the output error values in feed-forward network structure. By means of the program written in Delphi language, finding the best weights and optimal number of neurons in hidden layer have been attempted.

\section{EXPERIMENTS AND RESULTS}

In this study, diagnosis of epilepsy via EEG signals has been tried by using ANN and AIS. The public database constituted by Andrzejak and colleagues have been employed for this purpose. The obtained results by entering different number of iterations and by entering different number of neurons in hidden layer have been provided in Table I. A higher accuracy rate has been achieved by means of the used network parameters in training, namely with an increasing number of antibody and neurons in hidden layer. $93 \%, 96 \%$ accuracy rates have been obtained when the numbers of antibodies are 5, 10. As can be seen in Table I, near $100 \%$ accuracy has been obtained from the test results in case of the number of iterations 100 and mutation rate 0.05. Furthermore the average success rate has been obtained as $95 \%$ from all test scores.

The weights are updated and optimum weight values are tried to be determined with structure of back-propagation by means of performed hybrid structure with ANN and AIS. 


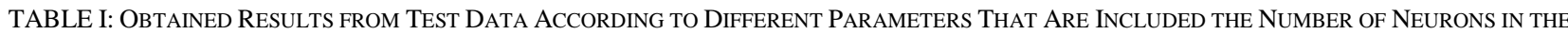
HIDDEN LAYER, ITERATION, ERROR RATE AND ANTIBODY THANKS BY HYBRID STRUCTURE OF ANN AND AIS.

\begin{tabular}{|c|r|c|c|c|c|c|c|c|c|c|c|c|c|}
\hline & $\begin{array}{r}\text { Neurons in } \\
\text { Hidden Layer }\end{array}$ & 2 & 4 & 6 & 2 & 4 & 6 & 2 & 4 & 6 & 2 & 4 & 6 \\
\cline { 2 - 11 } & Iteration & 50 & 50 & 50 & 100 & 100 & 100 & 50 & 50 & 50 & 100 & 100 & 100 \\
\cline { 2 - 11 } $\begin{array}{c}\text { Test Set } \\
\text { Parameters }\end{array}$ & Error Rate & 0,01 & 0,01 & 0,01 & 0,01 & 0,01 & 0,01 & 0,01 & 0,01 & 0,01 & 0,01 & 0,01 & 0,01 \\
\cline { 2 - 11 } & Antibody & 5 & 5 & 5 & 5 & 5 & 5 & 10 & 10 & 10 & 10 & 10 & 10 \\
\cline { 2 - 12 } & Mutation Rate & 0,01 & 0,01 & 0,01 & 0,05 & 0,05 & 0,05 & 0,01 & 0,01 & 0,01 & 0,05 & 0,05 & 0,05 \\
\hline \multirow{3}{*}{ Results } & Results 1 & $98 \%$ & $92 \%$ & $78 \%$ & $100 \%$ & $100 \%$ & $100 \%$ & $100 \%$ & $94 \%$ & $96 \%$ & $100 \%$ & $100 \%$ & $100 \%$ \\
\cline { 2 - 11 } & Results 2 & $62 \%$ & $100 \%$ & $94 \%$ & $100 \%$ & $100 \%$ & $96 \%$ & $62 \%$ & $100 \%$ & $100 \%$ & $100 \%$ & $100 \%$ & $100 \%$ \\
\cline { 2 - 11 } & Results 3 & $50 \%$ & $78 \%$ & $98 \%$ & $92 \%$ & $100 \%$ & $100 \%$ & $50 \%$ & $90 \%$ & $100 \%$ & $100 \%$ & $100 \%$ & $100 \%$ \\
\cline { 2 - 10 } & $82 \%$ & $100 \%$ & $100 \%$ & $100 \%$ & $100 \%$ & $100 \%$ & $100 \%$ & $100 \%$ & $100 \%$ & $100 \%$ & $100 \%$ & $100 \%$ \\
\hline
\end{tabular}

In this study, only the feed-forward network structure has been used in ANN model, AIS algorithm has only been used for updating the weights. Thus, the optimum weights have been obtained with less number of iterations by means of AIS while updating the optimal weights of network. It can be observed more quickly whether a person has epilepsy from EEG signals by means of the developed program that will take place in the future and will be computerized.

\section{ACKNOWLEDGEMENTS}

In the realization of this study, I thank Assist.Prof.Dr. Seral ÖZŞEN for share knowledge and experiences about the Artificial Immune Systems. Meanwhile, I also thank Assist.Prof.Dr. Gülay TEZEL for her assistance in conducting the EEG signals.

\section{REFERENCES}

[1] N. R. Sakthivel, B. B. Nair, V. Sugumaran, R. S. Rai, "Decision support system using artificial immune recognition system for fault classification of centrifugal pump," International Journal of Data Analysis Techniques and Strategies, vol. 3, no. 1, pp. 66-84, 2011.

[2] W. Zhao and C. E. Davis, "A modified artificial immune system based pattern recognition approach - an application to clinical diagnostics," Artificial Intelligence in Medicine, vol. 52, pp. 1-9, 2011.

[3] L. Guo, Y. Wu, T. Cao, W. Yan, and X. Shen, "Classification of mental task from eeg signals using immune feature weighted support vector machine," in Proc. $14^{\text {th }}$ Biennial IEEE Conference on Electromagnetic Field Computation (CEFC), 2010.

[4] A. Sengür, "An expert system based on principal component analysis, artificial immune system and fuzzy k-NN for diagnosis of valvular heart diseases," Computer in Biology and Medicine, vol. 38, issue 3, pp. 329-338, 2008.

[5] U. Aickelin, J. Greensmith, and J. Twycross, "Immune system approaches to intrusion detection - a review, artificial immune systems," Lecture Notes in Computer Science, vol. 3239/2004, pp. 316-329, 2004.

[6] A. A. Freitas and J. Timmis, "Revisiting the foundations of artificial immune systems: a problem-oriented perspective, artificial immune systems, artificial immune systems," Lecture Notes in Computer Science, vol. 2787/2003, pp. 229-241, doi: 10.1007/978-3540-45192-1_22, 2003.

[7] L. N. de Castro and J. Timmis, "An artificial immune network for multimodal function optimization," in Proc. the 2002 Congress on Evolutionary Computation CEC '02. , 2002, vol. 38, pp. 699-704.

[8] S. A. Hofmeyr and S. Forrest, "Architecture for an artificial immune system, evolutionary computation," Massachusetts Institute of Technology, vol. 8, no. 4, pp. 443-473, Winter 2000.
[9] C. A. Coello and N. C. Cortés, "Solving multi-objective optimization problems using an artificial immune system, genetic programming and evolvable machines," vol. 6, no. 2, pp. 163-190, 2005.

[10] P. L. Nunez and R. Srinivasan, Electric Fields of the Brain - The Neurophysics of EEG, Oxford University Press, New York, 2006.

[11] S. Sanei and J. Chambers, EEG Signal Processing, John Wiley \& Sons Ltd, West Sussex, 2007.

[12] S. Haykin, Neural Networks a Comprehensive Foundation, Prentice Hall, N. J., 1999.

[13] R. M. Satheesh Kumar, P. Asokan, S. Kumanan, "Artificial immune system-based algorithm for the unidirectional loop layout problem in a flexible manufacturing system," The International Journal of Advanced Manufacturing Technology, vol. 40, no. 5-6, pp. 553$565,2009$.

[14] W. Dong, N. Zhou, and J. C. Paul, "Robust tile-based texture synthesis using artificial immune system," Neural Computing \& Applications, vol. 18, no. 3, April, 2009.

[15] R. G. Andrzejak, K. Lehnertz, F. Mormann, C. Rieke, P. David, C. E. Elger, "Indications of nonlinear deterministic and finite-dimensional structures in time series of brain electrical activity: dependence on recording region and brain state," Phys Rev E Stat Nonlin Soft Matter Phys., 2001.

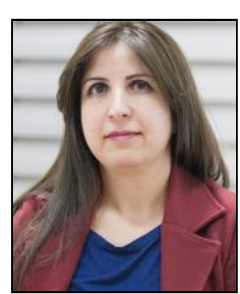

Sema Arslan was born in Diyadin, Turkey in 1974 She lived in Ankara until 2002. Afterwards, she came to Konya in order to sustain her education. She completed the graduate degree at the Department of Computer Systems and Teaching, Technical Education Faculty, Selcuk University in 2005. She completed master degree at the Department of Electronics and Computer Systems Education, Graduate School of Natural Sciences, Selcuk University in 2008. In this context, she searched and worked about ultrasound therapy in biomedical technologies. She began Ph.D. degree at the Department of Computer Engineering, Graduate School of Natural Sciences, Selcuk University in 2010. She is still studying her works in this department. Meanwhile, she surveys and search about biomedical signals in her $\mathrm{Ph}$.D. In that context, she tries to develop a new method in this field by using echo cancellation method. In addition to this, she performs her works with Prof. Dr. Inan Guler who works at Gazi University as second supervisor as well as Prof. Dr. Hakan Isik.

She worked as a computer operator at Gazi University between 2000 and 2002. Meanwhile, she has worked as a lecturer at Selcuk University since 2005. She used some Artificial Intelligence Techniques that are included Fuzzy Logic and Artificial Neural Network in her master thesis and these works were published some journals. She has also participated some international congress with her works that are included Artificial Intelligence, optimization techniques such as Particle Swarm Optimization and machine learning algorithms such as Genetic Algorithm and Artificial Immune Systems. 


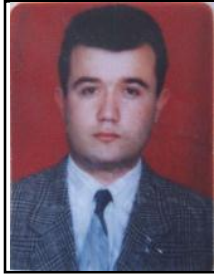

Hakan Isik was born in Adana, Turkey in 1968. He completed the graduate degree at the Department of Electronics and Computer Education, Technical Education Faculty, Gazi University in 1990. He completed master degree at the Department of Electronics and Computer Education, Graduate School of Natural Sciences, Gazi University in 1998 $\mathrm{He}$ began ph.D. degree at the Department of Electronics and Computer Education, Graduate School of Natural Sciences, Gazi University in 2002.

$\mathrm{He}$ is still studying her works as a professor at Department of Electronics Engineering, Technology Faculty, Selcuk University. He surveys and search about digital electronics, microprocessor, automatic control and PLC. He worked at Koc-Schindler lift industry between 1988 and 1990 as well as he worked at Sümer Holding between 1990 and 1994. Afterwards, he worked at Ondokuz Mayıs University between 1994 and 2002. Meanwhile, he has worked as a lecturer at Selcuk University since 2002. A lot of his work was published in various journals.

Prof. Isik performed as a program coordinator for four years. Afterwards, he has served head of the Department of Electronics, Selcuk University since 2004. In addition to these, he has been a member of faculty board and directors of faculty member of the board since 2006. Meanwhile, he had fulfilled some projects like thermal shoes that is supported by the Santez, Industrial Automation Staff Training Project (MEGEP) as an executive. 